\title{
THE CALABI (VERONESE) IMBEDDINGS AS INTEGRAL SUBMANIFOLDS OF $\mathbb{C} P^{2 n+1}$
}

\author{
D. E. BLAIR* \\ Department of Mathematics, Michigan State University, East Lansing, Michigan, U.S.A. \\ B. KORKMAZ \\ Department of Mathematics, Middle East Technical University, Ankara, Turkey \\ and L. VRANCKEN \\ Fachbereich Mathematik, Technische Universität Berlin, D-10623 Berlin, Germany
}

(Received 17 July, 1998)

\begin{abstract}
Considering odd-dimensional complex projective space as a complex contact manifold, one may ask which of the Calabi (Veronese) imbeddings can be positioned by a holomorphic congruence as integral submanifolds of the complex contact structure. It is first shown that when the first normal space is the whole normal space, this is impossible. It is also shown to be impossibile for a Calabi surface (complex dimension 2) in complex projective space of dimension 9 where one has both a first and second normal space. However when the complex dimension of the submanifold is odd and the whole normal space consists of the first and second normal spaces, then there is a holomorphic congruence positioning the Calabi imbedding as an integral submanifold of the complex contact structure.
\end{abstract}

1991 Mathematics Subject Classification. 53C15, 53C40, 53C55, 53C56.

1. Introduction. In [4] Calabi showed that up to holomorphic congruence there is a unique holomorphic imbedding of $\mathbb{C} P^{n}\left(\frac{4}{v}\right)$ into $\mathbb{C} P^{N}(4), N=\left(\begin{array}{c}n+v \\ v\end{array}\right)-1$ which does not lie in any totally geodesic complex projective space of lower dimension. Nakagawa and Ogiue [11] showed that the only full isometric immersions of positively curved complex space forms into positively curved complex space forms are local versions of these imbeddings. These imbeddings are known as the Calabi imbeddings or as the Veronese imbeddings, especially in the case $\mathbb{C} P^{2}(2) \longrightarrow \mathbb{C} P^{5}(4)$. For $n=1$ these imbeddings are called Calabi curves. Classically the Calabi imbeddings are given as follows. Let $\zeta_{1}, \ldots, \zeta_{n+1}$ be homogeneous coordinates for $\mathbb{C} P^{n}\left(\frac{4}{v}\right)$. The Calabi imbedding of $\mathbb{C} P^{n}\left(\frac{4}{v}\right)$ into $\mathbb{C} P^{N}(4)$, in terms of homogeneous coordinates for $\mathbb{C} P^{N}(4)$, is given by

$$
\left(\zeta_{1}, \ldots, \zeta_{n+1}\right) \longrightarrow\left(\zeta_{1}^{\nu}, \sqrt{v} \zeta_{1}^{\nu-1} \zeta_{2}, \ldots, \sqrt{\frac{v !}{a_{1} ! \cdots a_{n+1} !}} \zeta_{1}^{a_{1}} \cdots \zeta_{n+1}^{a_{n+1}}, \ldots, \zeta_{n+1}^{\nu}\right),
$$

where $\sum_{i=1}^{n+1} a_{i}=v$, the $a_{i}$ 's being non-negative integers.

The odd-dimensional complex projective spaces $\mathbb{C} P^{2 n+1}$ are well known examples of complex contact manifolds and in [1] the notion of a holomorphic Legendre curve was introduced. In [3] it was shown that the Calabi curves $\mathbb{C} P^{1}\left(\frac{4}{2 n+1}\right)$ can be

*Work supported in part by a Fulbright Research Scholarship at the Katholieke Universiteit Leuven, Belgium, 1997-98. 
brought by a holomorphic congruence into position as a holomorphic Legendre curve in $\mathbb{C} P^{2 n+1}(4)$. In contrast to this it was also shown in [3] that the Veronese surface $\mathbb{C} P^{2}(2)$ cannot be positioned as an integral submanifold of the complex contact structure on $\mathbb{C} P^{5}(4)$. Here we will show that the same argument proves that for $v=2$ there is no Calabi imbedding $\mathbb{C} P^{n}(2) \longrightarrow \mathbb{C} P^{\left(\begin{array}{c}n+2 \\ 2\end{array}\right)-1}(4)$ as an integral submanifold of the complex contact structure. When $v=3$ and $n$ is odd, $\left(\begin{array}{c}n+3 \\ 3\end{array}\right)-1$ is odd and we show that $\mathbb{C} P^{n}\left(\frac{4}{3}\right)$ can be realized as an integral submanifold of $\left.\mathbb{C} P^{\left(n^{n+3}\right.}\right)^{-1}(4)$. However one cannot expect this for $n$ even and we show that $\mathbb{C} P^{2}\left(\frac{4}{3}\right)$ cannot be positioned as an integral submanifold of the complex contact structure on $\mathbb{C} P^{9}(4)$.

2. Complex contact manifolds. A complex contact manifold is a complex manifold $M$ of odd complex dimension $2 n+1$ together with an open covering $\{\mathcal{U}\}$ of coordinate neighborhoods such that

(1) on each $\mathcal{U}$ there is a holomorphic 1 -form $\theta$ such that $\theta \wedge(d \theta)^{n} \neq 0$,

(2) on $\mathcal{U} \cap \mathcal{U}^{\prime} \neq \varnothing$ there is a non-vanishing holomorphic function $f$ such that $\theta^{\prime}=f \theta$.

The complex contact structure determines a non-integrable complex contact subbundle by the equation $\theta=0$ and a complementary vertical bundle which is typically assumed to be integrable.

It is important to note that the definition is analogous to that of a contact structure in the wider sense in view of the result [9] that for a compact complex manifold with $H^{1}\left(M, \mathbb{Z}_{n+1}\right)=0$, i.e. no $(n+1)$-torsion, a complex contact structure is given by a global 1 -form if and only if its first Chern class vanishes.

On the other hand if $M$ is a complex manifold with almost complex structure $J$, Hermitian metric $g$ and open covering by coordinate neighborhoods $\{\mathcal{U}\}$, it is called a complex almost contact metric manifold if it satisfies the following two conditions.

(1) In each $\mathcal{U}$ there exist 1 -forms $u$ and $v=u \circ J$ with dual vector fields $U$ and $V=-J U$ and $(1,1)$ tensor fields $G$ and $H=G J$ such that

$$
\begin{gathered}
G^{2}=H^{2}=-I+u \otimes U+v \otimes V, \\
G J=-J G, \quad G U=0, \quad g(X, G Y)=-g(G X, Y) .
\end{gathered}
$$

(2) On $\mathcal{U} \cap \mathcal{U}^{\prime} \neq \emptyset$, we have

$$
\begin{gathered}
u^{\prime}=a u-b v, \quad v^{\prime}=b u+a v, \\
G^{\prime}=a G-b H, \quad H^{\prime}=b G+a H,
\end{gathered}
$$

where $a$ and $b$ are functions with $a^{2}+b^{2}=1$.

A complex contact manifold admits a complex almost contact metric structure for which the local contact form $\theta$ is $u-i v$ to within a non-vanishing complex-valued function multiple and the local tensor fields $G$ and $H$ are related to $d u$ and $d v$ by

$$
d u(X, Y)=g(X, G Y)+(\sigma \wedge v)(X, Y), \quad d v(X, Y)=g(X, H Y)-(\sigma \wedge u)(X, Y)
$$

where $\sigma(X)=g\left(\nabla_{X} U, V\right), \nabla$ being the Levi-Civita connection of $g$ (see $\left.[\mathbf{5 , 8}]\right)$. We refer to a complex contact manifold with a complex almost contact metric structure satisfying these conditions as a complex contact metric manifold. 
Bearing in mind that the local contact form $\theta$ is $u-i v$ to within a non-vanishing complex-valued function multiple and since in the overlap $\mathcal{U} \cap \mathcal{U}^{\prime} \neq \emptyset, u^{\prime}=a u-b v$, $v^{\prime}=b u+a v$, we can define an integral submanifold as a submanifold whose tangent spaces belong to the complex contact subbundle, i.e. $u(X)=v(X)=0$ for any tangent vector $X$ or equivalently $\theta(X)=0$. If the submanifold is itself a complex submanifold, we call it a holomorphic integral submanifold. When a holomorphic integral submanifold has complex dimension 1, it is called a holomorphic Legendre curve [1]. It is well know in real contact geometry that the maximum dimension of an integral submanifold of a contact manifold of dimension $2 n+1$ is only $n$. Similarly it is easy to check that an integral submanifold of a complex contact manifold of complex dimension $2 n+1$ has real dimension at most $2 n$.

3. Complex contact structure on $\mathbb{C} P^{2 n+1}$. We will need a local coordinate expression for the complex contact structure on $\mathbb{C} P^{2 n+1}$. We will use homogeneous coordinates $\left(z_{1}, \ldots, z_{n+1}, w_{1}, \ldots, w_{n+1}\right)$. Then the complex contact structure is given by the holomorphic 1 -form

$$
\psi=\sum_{k=1}^{n+1}\left(z_{k} d w_{k}-w_{k} d z_{k}\right) .
$$

To give a little more detail we remark that the complex contact structure on $\mathbb{C} P^{2 n+1}$ is closely related to the Sasakian 3-structure on the unit sphere $S^{4 n+3}$ and to the quaternionic Kähler structure on quaternionic projective space. $\mathbb{C}^{2 n+2} \simeq \mathbb{H}^{n+1}$ has three almost complex structures $I, J, K$ which act on the position vector $\mathbf{x}$ as

$$
\begin{gathered}
I \mathbf{x}=i \mathbf{x}=\left(i z_{1}, \ldots, i z_{n+1}, i w_{1}, \ldots, i w_{n+1}\right), \\
J \mathbf{x}=\left(i \bar{w}_{1}, \ldots, i \bar{w}_{n+1},-i \bar{z}_{1}, \ldots,-i \bar{z}_{n+1}\right), \\
K \mathbf{x}=\left(\bar{w}_{1}, \ldots, \bar{w}_{n+1},-\bar{z}_{1}, \ldots,-\bar{z}_{n+1}\right) .
\end{gathered}
$$

The vector fields on $S^{4 n+3}$ given by $\xi_{1}=-I \mathbf{x}, \xi_{2}=-J \mathbf{x}, \xi_{3}=-K \mathbf{x}$ are the characteristic vector fields of three contact structures $\eta_{1}, \eta_{2}, \eta_{3}$ on $S^{4 n+3}$. In terms of the complex coordinates, the forms $\eta_{1}, \eta_{2}, \eta_{3}$ on $S^{4 n+3}$ are the restrictions of the following forms on $\mathbb{C}^{2 n+2}$, which we denote by the same letters.

$$
\begin{gathered}
\eta_{1}=-\frac{i}{2} \sum_{k=1}^{n+1}\left(z_{k} d \bar{z}_{k}-\bar{z}_{k} d z_{k}+w_{k} d \bar{w}_{k}-\bar{w}_{k} d w_{k}\right) \\
\eta_{3}+i \eta_{2}=\sum_{k=1}^{n+1}\left(z_{k} d w_{k}-w_{k} d z_{k}\right) .
\end{gathered}
$$

In [6] Ishihara and Konishi proved that if one of the contact structures of a manifold $\tilde{M}^{4 n+3}$ with a Sasakian 3-structure is regular, the base manifold $M$ of the induced fibration is a complex contact manifold. The structure is constructed as follows. If $\left(\phi_{1}, \xi_{1}, \eta_{1}, g\right)$, is the regular Sasakian structure, then $\phi_{1}$ and $g$ are 
projectable; (see for example [2] for notation). Let $X^{H}$ denote the horizontal lift of a vector field $X$ on $M$ with respect to the principal bundle connection defined by $\eta_{1}$. Then $J X=\pi_{*} \phi_{1} X^{H}$ and the projected metric form a Kähler structure on $M$. For a coordinate neighborhood $\mathcal{U}$ on $M$ and a local cross section $\tau$ of $\tilde{M}^{4 n+3}$ over $\mathcal{U}$, 1 -forms $u$ and $v$ and a tensor field $G$ defined on $\mathcal{U}$ by

$$
\begin{aligned}
& u(X) \circ \pi=\eta_{2}\left(\tau_{*} X\right), \quad v(X) \circ \pi=\eta_{3}\left(\tau_{*} X\right), \\
& G X=\pi_{*}\left(\phi_{2} \tau_{*} X-\eta_{1}\left(\tau_{*} X\right) \xi_{3}+\eta_{3}\left(\tau_{*} X\right) \xi_{1}\right)
\end{aligned}
$$

define the complex contact and complex almost contact structures on $M$. In the case of the Hopf fibration this is the standard Kähler structure on $\mathbb{C} P^{2 n+1}$ with the Fubini-Study metric of constant holomorphic curvature 4. With the Hopf fibration induced by $\xi_{1}, \psi=\eta_{3}+i \eta_{2}$ is a local expression for the complex contact structure on $\mathbb{C} P^{2 n+1}$.

We will also need the covariant derivatives of the structure tensors of the complex contact metric structure on $\mathbb{C} P^{2 n+1}$. These are the following, and we refer to $[7,10]$ for details.

$$
\begin{gathered}
\nabla_{X} U=-G X+\sigma(X) V, \quad \nabla_{X} V=-H X-\sigma(X) U . \\
\left(\nabla_{X} G\right) Y=g(X, Y) U-u(Y) X-g(X, J Y) V-v(Y) J X+\sigma(X) H Y . \\
\left(\nabla_{X} H\right) Y=g(X, Y) V-v(Y) X+g(X, J Y) U+u(Y) J X-\sigma(X) G Y .
\end{gathered}
$$

4. Submanifold theory. For a submanifold $M$ of a Riemannian manifold $(\tilde{M}, \tilde{g})$ we denote the induced metric by $g$. Then the Levi-Civita connection $\nabla$ of $g$ and the second fundamental form $\alpha$ are related to the ambient Levi-Civita connection $\tilde{\nabla}$ by

$$
\tilde{\nabla}_{X} Y=\nabla_{X} Y+\alpha(X, Y)
$$

For a normal vector field $\xi$ we denote by $A_{\xi}$ the corresponding Weingarten map and we denote the connection in the normal bundle by $\nabla^{\perp}$; in particular $A_{\xi}$ and $\nabla^{\perp}$ are defined by

$$
\tilde{\nabla}_{X} \xi=-A_{\xi} X+\nabla_{X}^{\perp} \xi
$$

and one readily has $\tilde{g}(\alpha(X, Y), \xi)=g\left(A_{\xi} X, Y\right)$. The Gauss equation is

$$
\tilde{R}(X, Y, Z, W)=R(X, Y, Z, W)+\tilde{g}(\alpha(X, Z), \alpha(Y, W))-\tilde{g}(\alpha(Y, Z), \alpha(X, W)) .
$$

Defining the covariant derivative of $\alpha$ by

$$
\left(\nabla^{\prime} \alpha\right)(X, Y, Z)=\nabla_{X}^{\perp} \alpha(Y, Z)-\alpha\left(\nabla_{X} Y, Z\right)-\alpha\left(Y, \nabla_{X} Z\right),
$$

the Codazzi equation is

$$
\left(R_{X Y} Z\right)^{\perp}=\left(\nabla^{\prime} \alpha\right)(X, Y, Z)-\left(\nabla^{\prime} \alpha\right)(Y, X, Z) .
$$


Finally for normal vector fields $\xi$ and $\eta$ the equation of Ricci-Kühn is

$$
\tilde{R}(X, Y, \xi, \eta)=R^{\perp}(X, Y, \xi, \eta)-g\left(\left[A_{\xi}, A_{\eta}\right] X, Y\right) .
$$

The span of the second fundamental form is called the first normal space and will be denoted by $v_{1}$. Let 'proj' denote projection to the orthogonal complement of $T M \oplus v_{1}$. Define $\beta(X, Y, Z)$ by

$$
\beta(X, Y, Z)=\operatorname{proj} \tilde{\nabla}_{X} \tilde{\nabla}_{Y} Z\left(=\operatorname{proj} \nabla_{X}^{\perp} \alpha(Y, Z)\right) .
$$

The span of $\beta$ is called the second normal space and will be denoted by $\nu_{2}$. Successively the higher normal spaces may be defined in this manner by taking higher order derivatives.

The meaning of the integer $v$ in the Calabi imbeddings $\mathbb{C} P^{n}\left(\frac{4}{v}\right) \longrightarrow \mathbb{C} P^{N}(4)$ is that there are $v-1$ normal spaces, each of maximal dimension; the dimension of the $r$ th normal space is $\frac{n}{r+1}\left(\begin{array}{c}n+r \\ r\end{array}\right)$. For a complex submanifold of a Kähler manifold one has $\alpha(X, J Y)=J \alpha(X, Y)$ and $\beta(X, Y, J Z)=J \beta(X, Y, Z)$. Moreover if the ambient space is a complex space form, the Codazzi equation gives that $\beta(X, Y, Z)$ is symmetric in $X, Y, Z$.

5. Calabi curves in $\mathbb{C} P^{2 n+1}(4)$. Before turning to our main topic, we mention for completeness the following result of [3].

THEOREM 1. There exists a holomorphic congruence of $\mathbb{C} P^{2 n+1}(4)$ that positions the Calabi curve $\mathbb{C} P^{1}\left(\frac{4}{2 n+1}\right)$ as a holomorphic Legendre curve in $\mathbb{C} P^{2 n+1}(4)$.

The imbedding is given explicitly as follows. For simplicity set $\alpha_{k}=\sqrt{\left(\begin{array}{c}2 n+1 \\ k-1\end{array}\right)}$; we position $\mathbb{C} P^{1}\left(\frac{4}{2 n+1}\right)$ by a holomorphic congruence of (1) as

$$
\begin{gathered}
\left(\zeta_{1}, \zeta_{2}\right) \longrightarrow \\
\left(\zeta_{1}^{2 n+1}, \ldots, \alpha_{k} \zeta_{1}^{2 n+2-k} \zeta_{2}^{k-1}, \ldots, \alpha_{n+1} \zeta_{1}^{n+1} \zeta_{2}^{n},\right. \\
\left.\zeta_{2}^{2 n+1}, \ldots,(-1)^{k-1} \alpha_{k} \zeta_{2}^{2 n+2-k} \zeta_{1}^{k-1}, \ldots,(-1)^{n} \alpha_{n+1} \zeta_{2}^{n+1} \zeta_{1}^{n}\right) .
\end{gathered}
$$

Then

$$
\begin{aligned}
\frac{\partial}{\partial \zeta_{1}} & =\sum_{k=1}^{m+1}\left(\alpha_{k}(2 n+2-k) \zeta_{1}^{2 n+1-k} \zeta_{2}^{k-1} \frac{\partial}{\partial z_{k}}+(-1)^{k-1} \alpha_{k}(k-1) \zeta_{2}^{2 n+2-k} \zeta_{1}^{k-2} \frac{\partial}{\partial w_{k}}\right) \\
\frac{\partial}{\partial \zeta_{2}} & =\sum_{k=1}^{m+1}\left(\alpha_{k}(k-1) \zeta_{1}^{2 n+2-k} \zeta_{2}^{k-2} \frac{\partial}{\partial z_{k}}+(-1)^{k-1} \alpha_{k}(2 n+2-k) \zeta_{2}^{2 n+1-k} \zeta_{1}^{k-1} \frac{\partial}{\partial w_{k}}\right)
\end{aligned}
$$

represent the tangent space and it suffices to show that $\psi\left(\frac{\partial}{\partial \zeta_{1}}\right)=\psi\left(\frac{\partial}{\partial \zeta_{2}}\right)=0$. 
6. On the higher dimensional Calabi imbeddings. In view of Theorem 1 , it is natural to ask if in addition to realizing the Calabi curves as holomorphic Legendre curves in $\mathbb{C} P^{2 n+1}(4)$, can the higher dimensional Calabi imbeddings be positioned as integral submanifolds of the complex contact structure on $\mathbb{C} P^{N}(4)$ for $N$ odd? In [3] it was shown that there is no holomorphic congruence of $\mathbb{C} P^{5}(4)$ that brings the Veronese surface $\mathbb{C} P^{2}(2)$ into position as a Legendre submanifold of the complex contact structure on $\mathbb{C} P^{5}(4)$. We generalize this as follows.

Theorem 2. Assume that $N=\left(\begin{array}{c}n+2 \\ 2\end{array}\right)-1$ is odd. There is no holomorphic congruence of $\mathbb{C} P^{N}(4)$ that brings the Calabi imbedding of $\mathbb{C} P^{n}(2)$ into position as an integral submanifold of the complex contact structure on $\mathbb{C} P^{N}(4)$.

Proof. Recall that the meaning of the condition $v=2$ is that the first normal space is the whole normal space. Suppose now that $\mathbb{C} P^{n}(2) \longrightarrow \mathbb{C} P^{N}(4)$ is an integral submanifold of the complex contact structure with second fundamental form $\alpha$. Since $u(X)=v(X)=0$ for any tangent vector $X$, the vector fields $U$ and $V$ are normal. Moreover $d u(X, Y)=0$ and hence from (2) or (3), $G X$ is normal for any tangent vector $X$. Now using (3) again we have

$$
0=X g(Y, U)=g\left(\tilde{\nabla}_{X} Y, U\right)-g(Y, G X)=g(\alpha(X, Y), U)
$$

and similarly $g(\alpha(X, Y), V)=0$. Thus $U$ and $V$ are orthogonal to both the tangent space and the first normal space, but the first normal space is the whole normal space, giving a contradiction.

Turning to $v=3$ we first give a non-existence result for $n=2$ and then a positive result for $n$ odd.

TheORem 3. There is no holomorphic congruence of $\mathbb{C} P^{9}(4)$ that brings the Calabi imbedding of $\mathbb{C} P^{2}\left(\frac{4}{3}\right)$ into position as an integral submanifold of the complex contact structure on $\mathbb{C} P^{9}(4)$.

Proof. We suppose that such an imbedding exists and seek a contradiction. Here $v=3$ and so we have both a first and second normal space. As in the previous proof $U$ and $V$ must belong to the second normal space. Also $G$ must map the tangent space into the normal space but a priori we do not know how the image of the tangent space lies relative to $\nu_{1}$ and $\nu_{2}$. Thus it is fundamental to consider $\tilde{g}(\alpha(X, Y), G Z)$ which, by virture of the equation $\tilde{\nabla}_{Z} U=-G Z+\sigma(Z) V$, is equal to $\tilde{g}(\beta(X, Y, Z), U)$. Using this and the Codazzi equation, we see that $\tilde{g}(\alpha(X, Y), G Z)$ is symmetric in $X, Y, Z$.

From the Gauss equation we have the following general formula.

$$
\begin{gathered}
\left(\frac{1}{v}-1\right)(g(Y, Z) g(X, W)-g(X, Z) g(Y, W) \\
+g(Y, J Z) g(X, J W)-g(X, J Z) g(Y, J W)-2 g(X, J Y) g(Z, J W)) \\
=\tilde{g}(\alpha(Y, Z), \alpha(X, W))-\tilde{g}(\alpha(X, Z), \alpha(Y, W)) .
\end{gathered}
$$


From this one readily obtains that $|\alpha(X, X)|^{2}=\frac{2(v-1)}{v}$, for any unit tangent vector $X$, and $|\alpha(X, Z)|^{2}=\frac{v-1}{v}$, for $Z$ orthogonal to $X$ and $J X$. Similarly one has the natural orthogonality relations: $\alpha(X, X) \perp \alpha(X, Z)$ for $X \perp Z ; \quad \alpha(X, X) \perp \alpha(Z, Z)$ for $X \perp Z, J Z$; etc. (cf. Nakagawa-Ogiue [11], equation (2.1)). Now consider an orthonormal basis of tangent vectors of the form $e_{1}, e_{2}, e_{3}=J e_{1}, e_{4}=J e_{2}$. In particular, setting $\alpha_{i j}=\alpha\left(e_{i}, e_{j}\right), \alpha_{11}, \alpha_{12}, \alpha_{22}, J \alpha_{11}, J \alpha_{12}, J \alpha_{22}$ is an orthogonal basis of the first normal space and $\left|\alpha_{11}\right|^{2}=\left|\alpha_{22}\right|^{2}=\frac{4}{3},\left|\alpha_{12}\right|^{2}=\frac{2}{3}$ (cf. Nakagawa-Ogiue [11], equation (2.1)).

Using the Codazzi equation and working at a point we have that $\tilde{g}\left(\nabla_{e_{j}}^{\perp} \alpha_{k l}, \alpha_{p q}\right)$ is symmetric in $j$ and $k$. Then differentiating the fact that the $\alpha_{i j}$ 's have constant length and using the results successively we obtain that $\nabla_{e_{j}}^{\perp} \alpha_{k l}$ is orthogonal to $v_{1}$ (cf. Nakagawa-Ogiue [11], equation (2.2)). Therefore $\beta(X, Y, Z)=\nabla_{X}^{\perp} \alpha(Y, Z)$ for $X, Y, Z$ belonging to the basis.

We can apply the same idea to $\beta$. From equation (2.4) of Nakagawa-Ogiue [11], we have that $\beta_{111}, \beta_{112}, \beta_{122}, \beta_{222}, J \beta_{111}, J \beta_{112}, J \beta_{122}, J \beta_{222}$ is an orthogonal basis of $\nu_{2}$ and $\left|\beta_{111}\right|^{2}=\left|\beta_{222}\right|^{2}=\frac{4}{3},\left|\beta_{112}\right|^{2}=\left|\beta_{122}\right|^{2}=\frac{4}{9}$. By virtue of the equation of RicciKühn, $\tilde{g}\left(\nabla_{e_{i}}^{\perp} \beta_{j k l}, \beta_{p q r}\right)=\tilde{g}\left(\nabla_{e_{i}}^{\perp} \nabla_{e_{j}}^{\perp} \alpha_{k l}, \beta_{p q r}\right)$ is symmetric in $i$ and $j$. Using this and the constancy of the lengths of the $\beta_{i j k}$ 's, we have that $\nabla_{e_{i}}^{\perp} \beta_{j k l}$ is orthogonal to $v_{2}$ (cf. Nakagawa-Ogiue [11], equation (2.7)).

Using this last property and equations (3) and (4), differentiation of $\tilde{g}(\alpha(X, Y), G Z)=\tilde{g}(\beta(X, Y, Z), U)$ yields

$$
\tilde{g}(\beta(W, X, Y), G Z)+\tilde{g}(\beta(X, Y, Z), G W)=-\tilde{g}(\alpha(X, Y), G \alpha(Z, W))
$$

from which we deduce that

$$
\begin{aligned}
& \tilde{g}(\beta(W, Y, Z), G X)+\tilde{g}(\beta(X, Y, Z), G W)=-\tilde{g}(\alpha(Y, Z), G \alpha(X, W)), \\
& -\tilde{g}(\beta(Z, Y, W), G X)-\tilde{g}(\beta(X, Y, W), G Z)=-\tilde{g}(\alpha(X, Z), G \alpha(Y, W)) .
\end{aligned}
$$

Adding these three equations yields

$$
\tilde{g}(\beta(X, Y, Z), G W)=-\frac{1}{2}\left(\sum_{X Y Z} \tilde{g}(\alpha(X, Y), G \alpha(Z, W)),\right.
$$

where the sum is the cyclic sum on $X Y Z$. In particular we see that

$$
\tilde{g}(\beta(X, X, X), G X)=0 .
$$

Differentiating this with respect to a vector field $Y$ and using (4), we have

$$
\begin{gathered}
\tilde{g}\left(\nabla_{Y}^{\perp} \beta(X, X, X), G X\right)+\tilde{g}(\beta(X, X, X), g(X, Y) U+g(X, J Y) V) \\
+\tilde{g}(\beta(X, X, X), G \alpha(X, Y))=0 .
\end{gathered}
$$

If $Y$ is orthogonal to both $X$ and $J X$, then, since $G X$ is normal, we have from our discussion of $\beta_{i j k}$ and $\nabla_{e_{i}}^{\perp} \beta_{j k l}$ above that

$$
\tilde{g}(\beta(X, X, X), G \alpha(X, Y))=0 .
$$


If $Y=X$ and $X$ is unit, then bearing in mind that $\tilde{g}(\alpha(X, X), G X)=\tilde{g}(\beta(X, X, X), U)$, we see in a similar manner that $\nabla_{X}^{\perp} \beta(X, X, X)=-\alpha(X, X)$. From this it follows that

$$
\tilde{g}(\beta(X, X, X), G \alpha(X, X))=0
$$

Since, in fact, (7) is valid for all $X$, linearization gives

$$
\tilde{g}(\beta(X, X, Y), G \alpha(X, X))=0
$$

for $Y \perp X, J X$. Also the above equations (5) through (8) hold for $G$ replaced by $H$. Continuing, differentiation of (7) with respect to $Y \perp X, J X$ and consideration of the projections of $G \alpha(X, X)$ to the tangent space and to $v_{1}$ and $v_{2}$ gives

$$
\tilde{g}(\beta(X, X, X), G \beta(X, X, Y))=0
$$

and a similar result for $H$.

To analyse $\tilde{g}(\alpha(X, Y), G Z)$ we introduce a special basis of tangent vectors. Regarding $\tilde{g}(\alpha(X, X), G X)$ as a function on the unit tangent bundle of $\mathbb{C} P^{2}\left(\frac{4}{3}\right)$, which is compact, it attains its maximum at a unit vector $e_{1}$ at a point $p$. If this maximum is zero, then $\tilde{g}(\alpha(X, X), G X)$ vanishes identically and therefore linearization would imply that $U$ is orthogonal to both the first and second normal spaces, a contradiction. It now follows that $e_{1}$ is an eigenvector of the Weingarten map $A_{G e_{1}}$ with positive eigenvalue which we denote by $a_{1}$. Since for Kähler submanifolds, the Weingarten maps anti-commute with $J, e_{3}=J e_{1}$ is also an eigenvector with eigenvalue $-a_{1}$. Now let $e_{2}$ be another eigenvector with eigenvalue $a_{2}$ and $e_{4}=J e_{2}$. From the symmetry of $\tilde{g}(\alpha(X, Y), G Z)=g\left(A_{G Z} X, Y\right)$, the equations $A_{G e_{3}}=$ $-A_{J G e_{1}}=-J A_{G e_{1}}$ and similar equations for $A_{G e_{4}}$, we have that these Weingarten maps are given by the following matrices.

$$
\begin{array}{ccc}
A_{G e_{1}}=\left(\begin{array}{cccc}
a_{1} & 0 & 0 & 0 \\
0 & a_{2} & 0 & 0 \\
0 & 0 & -a_{1} & 0 \\
0 & 0 & 0 & -a_{2}
\end{array}\right), & A_{G e_{2}}=\left(\begin{array}{cccc}
0 & a_{2} & 0 & 0 \\
a_{2} & a_{3} & 0 & a_{4} \\
0 & 0 & 0 & -a_{2} \\
0 & a_{4} & -a_{2} & -a_{3}
\end{array}\right), \\
A_{G e_{3}}=\left(\begin{array}{cccc}
0 & 0 & -a_{1} & 0 \\
0 & 0 & 0 & -a_{2} \\
-a_{1} & 0 & 0 & 0 \\
0 & -a_{2} & 0 & 0
\end{array}\right), & A_{G e_{4}}=\left(\begin{array}{cccc}
0 & 0 & 0 & -a_{2} \\
0 & a_{4} & -a_{2} & -a_{3} \\
0 & -a_{2} & 0 & 0 \\
-a_{2} & -a_{3} & 0 & -a_{4}
\end{array}\right) .
\end{array}
$$

From the relation $\tilde{g}(\beta(X, Y, Z), U)=\tilde{g}(\alpha(X, Y), G Z)=g\left(A_{G Z} X, Y\right)$ we see that

$$
U=\frac{3}{4} a_{1} \beta_{111}+\frac{9}{4} a_{2} \beta_{122}+\frac{3}{4} a_{3} \beta_{222}+\frac{3}{4} a_{4} J \beta_{222}
$$

and $U$ being unit gives

$$
a_{1}^{2}+3 a_{2}^{2}+a_{3}^{2}+a_{4}^{2}=\frac{4}{3} .
$$


We now introduce the following notation.

$$
\begin{aligned}
& b_{1}=\tilde{g}\left(G \alpha_{11}, \alpha_{12}\right), \quad b_{4}=\tilde{g}\left(G \alpha_{11}, J \alpha_{22}\right), \\
& b_{2}=\tilde{g}\left(G \alpha_{11}, J \alpha_{12}\right), \quad b_{5}=\tilde{g}\left(G \alpha_{12}, \alpha_{22}\right) \text {, } \\
& b_{3}=\tilde{g}\left(G \alpha_{11}, \alpha_{22}\right), \quad b_{6}=\tilde{g}\left(G \alpha_{12}, J \alpha_{22}\right) \text {, } \\
& c_{1}=\tilde{g}\left(G e_{1}, \beta_{111}\right), \quad c_{9}=\tilde{g}\left(G e_{2}, \beta_{111}\right), \\
& c_{2}=\tilde{g}\left(G e_{1}, J \beta_{111}\right), \quad c_{10}=\tilde{g}\left(G e_{2}, J \beta_{111}\right), \\
& c_{3}=\tilde{g}\left(G e_{1}, \beta_{112}\right), \quad c_{11}=\tilde{g}\left(G e_{2}, \beta_{112}\right) \text {, } \\
& c_{4}=\tilde{g}\left(G e_{1}, J \beta_{112}\right), \quad c_{12}=\tilde{g}\left(G e_{2}, J \beta_{112}\right), \\
& c_{5}=\tilde{g}\left(G e_{1}, \beta_{122}\right), \quad c_{13}=\tilde{g}\left(G e_{2}, \beta_{122}\right), \\
& c_{6}=\tilde{g}\left(G e_{1}, J \beta_{122}\right), \quad c_{14}=\tilde{g}\left(G e_{2}, J \beta_{122}\right) \text {, } \\
& c_{7}=\tilde{g}\left(G e_{1}, \beta_{222}\right), \quad c_{15}=\tilde{g}\left(G e_{2}, \beta_{222}\right) \text {, } \\
& c_{8}=\tilde{g}\left(G e_{1}, J \beta_{222}\right), \quad c_{16}=\tilde{g}\left(G e_{2}, J \beta_{222}\right), \\
& d_{1}=\tilde{g}\left(G \alpha_{11}, \beta_{111}\right), \quad d_{13}=\tilde{g}\left(G \alpha_{12}, \beta_{122}\right), \\
& d_{2}=\tilde{g}\left(G \alpha_{11}, J \beta_{111}\right), \quad d_{14}=\tilde{g}\left(G \alpha_{12}, J \beta_{122}\right), \\
& d_{3}=\tilde{g}\left(G \alpha_{11}, \beta_{112}\right), \quad d_{15}=\tilde{g}\left(G \alpha_{12}, \beta_{222}\right) \text {, } \\
& d_{4}=\tilde{g}\left(G \alpha_{11}, J \beta_{112}\right), \quad d_{16}=\tilde{g}\left(G \alpha_{12}, J \beta_{222}\right), \\
& d_{5}=\tilde{g}\left(G \alpha_{11}, \beta_{122}\right), \quad d_{17}=\tilde{g}\left(G \alpha_{22}, \beta_{111}\right), \\
& d_{6}=\tilde{g}\left(G \alpha_{11}, J \beta_{122}\right), \quad d_{18}=\tilde{g}\left(G \alpha_{22}, J \beta_{111}\right), \\
& d_{7}=\tilde{g}\left(G \alpha_{11}, \beta_{222}\right), \quad d_{19}=\tilde{g}\left(G \alpha_{22}, \beta_{112}\right), \\
& d_{8}=\tilde{g}\left(G \alpha_{11}, J \beta_{222}\right), \quad d_{20}=\tilde{g}\left(G \alpha_{22}, J \beta_{112}\right), \\
& d_{9}=\tilde{g}\left(G \alpha_{12}, \beta_{111}\right), \quad d_{21}=\tilde{g}\left(G \alpha_{22}, \beta_{122}\right), \\
& d_{10}=\tilde{g}\left(G \alpha_{12}, J \beta_{111}\right), \quad d_{22}=\tilde{g}\left(G \alpha_{22}, J \beta_{122}\right), \\
& d_{11}=\tilde{g}\left(G \alpha_{12}, \beta_{112}\right), \quad d_{23}=\tilde{g}\left(G \alpha_{22}, \beta_{222}\right), \\
& d_{12}=\tilde{g}\left(G \alpha_{12}, J \beta_{112}\right), \quad d_{24}=\tilde{g}\left(G \alpha_{22}, J \beta_{222}\right), \\
& f_{1}=\tilde{g}\left(G \beta_{111}, \beta_{112}\right), \quad f_{7}=\tilde{g}\left(G \beta_{112}, \beta_{122}\right), \\
& f_{2}=\tilde{g}\left(G \beta_{111}, J \beta_{112}\right), \quad f_{8}=\tilde{g}\left(G \beta_{112}, J \beta_{122}\right), \\
& f_{3}=\tilde{g}\left(G \beta_{111}, \beta_{122}\right), \quad f_{9}=\tilde{g}\left(G \beta_{112}, \beta_{222}\right), \\
& f_{4}=\tilde{g}\left(G \beta_{111}, J \beta_{122}\right), \quad f_{10}=\tilde{g}\left(G \beta_{112}, J \beta_{222}\right), \\
& f_{5}=\tilde{g}\left(G \beta_{111}, \beta_{222}\right), \quad f_{11}=\tilde{g}\left(G \beta_{122}, \beta_{222}\right), \\
& f_{6}=\tilde{g}\left(G \beta_{111}, J \beta_{222}\right), \quad f_{12}=\tilde{g}\left(G \beta_{122}, J \beta_{222}\right) \text {. }
\end{aligned}
$$

Applying equation (5), i.e. $\tilde{g}\left(G e_{i}, \beta_{j k l}\right)=-\frac{1}{2}\left(\sum_{j k l} \tilde{g}\left(\alpha_{j k}, G \alpha_{l i}\right)\right)$, we have

$$
\begin{aligned}
& \begin{array}{l}
c_{1}=0, \\
c_{2}=0, \\
c_{3}=-\frac{1}{2} b_{1}, \\
c_{4}=-\frac{1}{2} b_{2},
\end{array} \\
& c_{5}=-\frac{1}{2} b_{3}, \\
& c_{6}=-\frac{1}{2} b_{4} \text {, } \\
& c_{7}=-\frac{3}{2} b_{5}, \\
& c_{8}=-\frac{3}{2} b_{6}, \\
& c_{9}=\frac{3}{2} b_{1}, \\
& c_{10}=\frac{3}{2} b_{2} \text {, } \\
& c_{11}=\frac{1}{2} b_{3} \text {, } \\
& c_{13}=\frac{1}{2} b_{5}, \\
& c_{14}=\frac{1}{2} b_{6} \text {, } \\
& c_{15}=0 \text {, } \\
& c_{12}=\frac{1}{2} b_{4}, \\
& c_{16}=0 \text {. }
\end{aligned}
$$


Similarly from equations (6), (7) and (8) we readily have $d_{1}=d_{2}=d_{3}$ $=d_{4}=d_{9}=d_{10}=d_{15}=d_{16}=d_{21}=d_{22}=d_{23}=d_{24}=0$ and from (9) we have $f_{1}=f_{2}=f_{11}=f_{12}=0$.

Recall that $p$ and $e_{1}$ were the point and unit tangent vector where $\tilde{g}(\alpha(X, X), G X)$ regarded as a function, say $f(X)$, on the unit tangent bundle attained its maximum. For any unit tangent vector $Y$ at $p$, let $\gamma(t)$ be the geodesic in $\mathbb{C} P^{2}\left(\frac{4}{3}\right)$ with $\gamma(0)=p$ and $\gamma^{\prime}(0)=Y$. Let $X(t)$ be the parallel vector field along $\gamma$ with $X(0)=e_{1}$. Then

$$
0=\left.\frac{d}{d t} f(X(t))\right|_{t=0}=\tilde{g}(\beta(Y, X, X), G X) .
$$

Thus taking $Y$ to be orthogonal to $e_{1}$ we see that $c_{3}=c_{4}=0$ and hence that $b_{1}=b_{2}=0$.

Now one may write out $G e_{i}, G \alpha_{i j}, G \beta_{i j k}$ as necessary; for example, bearing in mind the coefficients known to be zero so far,

$$
G \alpha_{11}=-a_{1} e_{1}+\frac{3}{4} b_{3} \alpha_{22}+\frac{3}{4} b_{4} J \alpha_{22}+\frac{9}{4} d_{5} \beta_{122}+\frac{9}{4} d_{6} J \beta_{122}+\frac{3}{4} d_{7} \beta_{222}+\frac{3}{4} d_{8} J \beta_{222}
$$

Then expanding $0=\tilde{g}\left(G \alpha_{11}, G \alpha_{22}\right)=a_{1} a_{2}$ we have $a_{2}=0$. Similarly using $f_{11}=f_{12}=0$ we have $0=\tilde{g}\left(G \alpha_{11}, G \beta_{222}\right)=a_{1} c_{7}$ and also $0=\tilde{g}\left(G \alpha_{11}, G J \beta_{222}\right)=a_{1} c_{8}$; thus since $a_{1} \neq 0$ we have $c_{7}=c_{8}=0$. In turn $b_{5}=b_{6}=0$ and $c_{13}=c_{14}=0$.

Writing out $G e_{1}$, we now have

$$
G e_{1}=\frac{3}{4} a_{1} \alpha_{11}+\frac{9}{4} c_{5} \beta_{122}+\frac{9}{4} c_{6} J \beta_{122} .
$$

Since $G^{2}=-I$ on tangent vectors and expanding $G \alpha_{11}$, etc. we have

$$
\begin{gathered}
-e_{1}=\frac{3}{4} a_{1}\left(-a_{1} e_{1}+\frac{3}{4} b_{3} \alpha_{22}+\frac{3}{4} b_{4} J \alpha_{22}+\text { terms in } v_{2}\right), \\
-\frac{9}{8} b_{3}\left(-c_{5} e_{1}-c_{6} e_{3}-\frac{3}{4} d_{5} \alpha_{11}-\frac{3}{4} d_{6} J \alpha_{11}-\frac{3}{2} d_{13} \alpha_{12}-\frac{3}{2} d_{14} J \alpha_{12}+\text { terms in } v_{2}\right), \\
-\frac{9}{8} b_{4}\left(-c_{6} e_{1}+c_{5} e_{3}-\frac{3}{4} d_{6} \alpha_{11}+\frac{3}{4} d_{5} J \alpha_{11}-\frac{3}{2} d_{14} \alpha_{12}+\frac{3}{2} d_{13} J \alpha_{12}+\text { terms in } v_{2}\right) .
\end{gathered}
$$

From the $\alpha_{22}$ and $J \alpha_{22}$ components we see that $b_{3}=b_{4}=0$. Therefore we now have that all $c_{i}$ 's vanish. Then from the $e_{1}$ component we see that $a_{1}^{2}=\frac{4}{3}$ and hence from (10) we have $a_{3}=a_{4}=0$. Thus with $a_{2}=a_{3}=a_{4}=0$ and all $c_{i}=0$, we have that $G e_{2}=0$, a contradiction.

Theorem 4. When $v=3$ and $n$ is odd, $N=\left(\begin{array}{c}n+3 \\ 3\end{array}\right)-1$ is odd and there exists a holomorphic congruence of $\mathbb{C} P^{N}(4)$ that brings the Calabi imbedding of $\mathbb{C} P^{n}\left(\frac{4}{3}\right)$ into position as an integral submanifold of the complex contact structure on $\mathbb{C} P^{N}(4)$.

Proof. Suppose that $n=2 p+1$ and consider a column of $z_{h}$ 's and of $w_{h}$ 's, $(h=1, \ldots, P), \quad P=\frac{1}{3}(p+1)(p+2)(2 p+3)=\frac{1}{2}(N+1)$. For the first $p+1$, set $z_{h}=\zeta_{h}^{3}$ and $w_{h}=-\zeta_{p+h+1}^{3}$. 
For the next $(p+1)(2 p+1)$ indices form $p+1$ sets of the form $\sqrt{3} \zeta_{j}^{2} \zeta_{k}$ in the $z$-column, first taking all the $\zeta_{1}^{2}$, s times the remaining $\zeta_{k}$ in order, then all the $\zeta_{2}^{2}$, s times the $\zeta_{k}, k \neq 2$ in order, etc. through the $\zeta_{p+1}^{2}$. Corresponding to these terms in the $w$ column use $\pm \sqrt{3} \zeta_{j}^{2} \zeta_{k}, j$ running through $p+2, \ldots, 2 p+2$ and $k$ running through the variables $\zeta_{k}, k \neq j$, first with $k \in\{p+2, \ldots, 2 p+2\}$ and the sign negative and then with $k \in\{1, \ldots, p+1\}$ and the sign positive.

The last $\frac{1}{3} p(p+1)(2 p+1)$ terms in the $z$ column are of the form $\sqrt{6} \zeta_{i} \zeta_{j} \zeta_{k}$, $(i<j<k)$, and taken as follows. First order the pairs $i j$ as

$$
12,13,23,14,24,34, \ldots, 1(p+1), \ldots, p(p+1) .
$$

Each pair $\sqrt{6} \zeta_{i} \zeta_{j}$ is multiplied successively by $\zeta_{k},(k=j+1,2 p+2)$. For the $w$ column the terms are of the form $\pm \sqrt{6} \zeta_{i} \zeta_{j} \zeta_{k}$ with the pairs $i j$ ordered as $(p+2)(p+3),(p+2)(p+4), \ldots,(2 p+1)(2 p+2)$. Then each pair is multiplied successively by $-\zeta_{k}, k=j+1,, 2 p+2$ and then by $+\zeta_{k}, k=1, p+1$.

The proof now is to evaluate the complex contact form $\psi$ on $\frac{\partial}{\partial \zeta_{i}}$. The computation is tedious but straightforward and the result is that

$$
\psi\left(\frac{\partial}{\partial \zeta_{l}}\right)=\sum_{k=1}^{P}\left(z_{k} \frac{\partial w_{k}}{\partial \zeta_{l}}-w_{k} \frac{\partial z_{k}}{\partial \zeta_{l}}\right)=0,
$$

as desired.

We end with a question for further study. For $N$ odd, which of the Calabi imbeddings can be realized as an integral submanifold of the complex contact structure on $\mathbb{C} P^{N}(4)$ ? Must $n$ also be odd?

\section{REFERENCES}

1. C. Baikoussis, D. E. Blair and F. Gouli-Andreou, Holomorphic Legendre curves in the complex Heisenberg group, Bull. Inst. Acad. Sinica 26 (1998), 179-194.

2. D. E. Blair, Contact manifolds in Riemannian geometry, Lecture Notes in Mathematics, No. 509 (Springer-Verlag, 1976).

3. D. E. Blair, F. Dillen, L. Verstraelen and L. Vrancken, Calabi curves as holomorphic Legendre curves and Chen's inequality, Kyungpook Math. J. 35 (1996), 407-416.

4. E. Calabi, Isometric imbeddings of complex manifolds, Ann. of Math. 58 (1953), 1-23.

5. B. J. Foreman, Variational problems on complex contact manifolds with applications to twistor space theory, Thesis (Michigan State University, 1996).

6. S. Ishihara and M. Konishi, Real contact 3-structure and complex contact structure, Southeast Asian Bull. Math. 3 (1979), 151-161.

7. S. Ishihara and M. Konishi, Complex almost contact manifolds, Kodai Math. J. 3 (1980), 385-396.

8. S. Ishihara and M. Konishi, Complex almost contact structures in a complex contact manifold, Kodai Math. J. 5 (1982), 30-37.

9. S. Kobayashi, Remarks on complex contact manifolds, Proc. Amer. Math. Soc. 10 (1959), 164-167.

10. B. Korkmaz, Curvature and normality of complex contact manifolds, Ph.D. Thesis (Michigan State University, 1997).

11. H. Nakagawa and K. Ogiue, Complex space forms immersed in complex space forms, Trans. Amer. Math. Soc. 219 (1976), 289-297. 